\title{
LA CATEGORÍA ITINERARIOS CULTURALES Y SU SIGNIFICADO EN LA EVOLUCIÓN TEÓRICO-CONCEPTUAL DEL PATRIMONIO CULTURAL
}

\author{
THE CATEGORY CULTURAL ROUTES AND ITS MEANING IN THE THEORETICAL EVOLUTION OF THE \\ CONCEPT CULTURAL HERITAGE
}

ALBERTO MARTORELL CARREÑO

\begin{abstract}
RESUMEN
La evolución del concepto de patrimonio cultural tiene en la inclusión de la categoría itinerario cultural, un cambio que, si bien sigue una tendencia marcada por otros avances previos (como los conceptos de paisaje cultural y patrimonio seriado), una magnitud que podría inclusive calificarse de un cambio de paradigma. Lo demuestran grandes proyectos como el del Qhapaq Ñan, la Ruta de la Seda, y otros similares que vienen siendo promovidos desde la UNESCO y que involucran una escala geográfica y una diversidad tales que casi no se podrían haber imaginado previamente. En el presente artículo buscamos presentar los pasos más significativos en ese proceso inclusivo que viene siendo materia de gran interés académico y cultural en los últimos años. Hay, sin embargo, una serie de vacíos e imprecisiones todavía en el concepto, sobre los que reflexionamos también en el presente artículo. Exponemos la necesidad de generar un proceso de evaluación de los avances hasta ahora realizados y de reforzar algunos de los valores que el nuevo concepto pone en evidencia.
\end{abstract}

\section{PALABRAS CLAVE}

Itinerarios culturales, itinerarios patrimoniales, patrimonio mundial, conectividad, interelación.

\begin{abstract}
The inclusion of the category cultural heritage within the conceptual framework of the cultural heritage implies a deep change that could even be considered the arising of a new paradigm, despite of the fact that it follows a tendency previously marked by other inclusions (i.e. cultural landscape and serial heritage). Huge projects such as the Qhapaq Nan and the Silk Road and others promoted by UNESCO are a demonstration of this statement. They imply a geographic scope and diversity that would even not be in the previous imagination. In this article, we follow the most important steps in this inclusive process which is arising the academic and cultural interest for the last years. There are, nevertheless, some conceptual gaps and vagueness which we analyze in this article. We make evident the necessity to generate an evaluation process of the progress made until now in this issue and to strengthen some values that the concept cultural routes itself makes evident.
\end{abstract}

\section{KEY WORDS}

Cultural routes, heritage routes, world heritage, connectivity, interrelationship. 


\section{Introducción}

Cuando se analizaba la inclusión del Camino de Santiago en España, en 1993, primer caso de una ruta de comunicación presentado ante la UNESCO para su inscripción en la Lista del Patrimonio Mundial (LPM), el Consejo Internacional de Monumentos y Sitios (ICOMOS, por sus siglas en inglés) en su informe sobre el caso (ICOMOS, 1993) formuló el siguiente "comentario adicional":

\begin{abstract}
Those sites and monuments already on the World Heritage List are individual monuments, cohesive ensembles, or thematic groups. The ensembles are nucleated rather than linear, which is the case of the Route. The two "linear" monuments already on the List (the Great Wall of China and Hadrian's Wall) are both continuous military structures with a clear identity. The Route of St James differs from all the existing properties on the List in that it is essentially a communications route, with the structures and settlements associated with it, and as such is difficult to evaluate according to the criteria set out in the Operational Guidelines for the Convention. It is therefore of the opinion that a working group should be set up without delay to consider what changes and/or additions to the Operational Guidelines might be needed to accommodate properties of this kind. This proposal has been accepted by the Spanish Government, which is funding an expert meeting, to be held in early 1994. (pp. 62-63)
\end{abstract}

Ponían así los expertos informantes de ICOMOS, en evidencia, que se encontraban ante una forma de patrimonio que hasta el momento les había sido desconocida, y consideraban necesario promover su análisis y mayor reflexión. La reunión a cuya aceptación se hace referencia en la cita anterior efectivamente se realizó en Madrid el año de 1994. Algunas de las observaciones que en esa ocasión se formularon se han mantenido en el núcleo conceptual de la materia hasta nuestros días, aunque no en todos los casos podemos considerar que hayan sido logros inalterables.

Entre los aportes más significativos de esa reunión podemos mencionar la referencia al entendimiento mutuo entre los pueblos, a una aproximación plural a la historia y a la cultura de paz (UNESCO, 1994) que, si bien es cierto no tienen la forma de fórmulas conceptuales, sí permiten vislumbrar la importancia del tipo de bienes sobre el que venimos tratando. También se afirmó que este concepto se basa en el movimiento de personas, los encuentros y el diálogo, los intercambios y la interfertilización, que se realizan tanto en la dimensión espacial como en la temporal.
En estos últimos elementos podemos encontrar las líneas de pensamiento que otorgan, correctamente presentados, su peculiaridad conceptual y técnica al concepto de itinerarios culturales. En efecto, ubican primero al tema dentro de una de las divisiones que, años después, propondría ICOMOS en su clasificación del patrimonio mundial (ICOMOS, 2004). En dicho documento, ICOMOS hace un ejercicio de análisis de los bienes hasta ese momento inscritos en la Lista del Patrimonio Mundial. Propone tres marcos de clasificación: uno tipológico, uno temático y uno cronológico-regional.

El marco temático incluye las siguientes divisiones:

1. Asociaciones culturales, dentro de la que consideran a su vez tres subdivisiones: interacción humana en sociedad, asociaciones culturales y simbólicas y ramas del conocimiento.

2. Expresiones de la creatividad, que a su vez se subdivide en monumentos, grupos de edificios y sitios.

3. Respuestas espirituales: dentro de este tema se proponen los subtemas: sistemas de creencias antiguos y de pueblos indígenas, hinduismo y religiones relacionadas, budismo, confucianismo, taoísmo y sintoísmo, judaísmo, cristiandad; e Islam.

4. Utilización de los recursos naturales: que comprenden la agricultura y producción alimentaria, la minería e industrias extractivas, y los sistemas de manufactura.

5. Movimiento de personas: y dentro de él una división que no nos resulta del todo adecuada: migración, nomadismo y esclavismo; y rutas y sistemas de transporte.

6. Desarrollo de tecnologías.

Entendiéndose que los itinerarios culturales tenían, desde las discusiones iniciales en 1994, una identificación con el tema movimiento de personas, resulta entonces que una pista válida para ahondar en el concepto tratado apunta a comprender lo que este tema implica.

Sin embargo, antes de que el término itinerarios culturales se usara en el ámbito del patrimonio, ya había sido materia de otros contextos con enfoques distintos al que venimos exponiendo, y que podríamos tipificar como netamente patrimonial. Es el caso del llamado Programa Europeo de Itinerarios Culturales. 


\section{Los "itinerarios culturales europeos" como an- tecedente y variante terminológica}

En realidad, cuando el Camino de Santiago de Compostela se presenta para su inclusión en la Lista del Patrimonio Mundial (LPM), su condición de itinerario cultural había sido ya reconocida por el Consejo de Europa dentro de su programa "itinerarios culturales". Tal es así que el ya citado informe de ICOMOS (1993) hace referencia a ese hecho, tomando nota de que el llamado "Camino Francés" no incluye la totalidad del Camino de Santiago, sino que este se extiende por otros territorios europeos. Insta, sobre la anterior base, a que los Estados europeos por los que el antiguo Camino Jacobeo discurre, estudien también medidas para su declaración. Lo hizo únicamente Francia (que bajo el nombre "Caminos de Santiago de Compostela en Francia" lo inscribe en la LPM en 1998), y bajo una modalidad bastante distinta que no necesariamente reconoce la dimensión unitaria que un itinerario cultural verdaderamente posee.

El Consejo de Europa, dentro de su programa "Itinerarios Culturales Europeos" declaró como primera ruta continental a "Los Caminos de Peregrinaje a Santiago de Compostela", comprendiendo la extensión del camino a su paso por Bélgica, Francia, Alemania, Italia, Luxemburgo, España y Suiza.

Resulta interesante la descripción que sobre estos Caminos de Peregrinaje se hace en la página web del Consejo de Europa (s.f), pues dada la magnitud patrimonial de estas rutas, se da una descripción que propiamente corresponde a la de un itinerario en términos patrimoniales:

\footnotetext{
Pilgrims really did gain an incomparable cultural experience. They could discover various new customs, languages and ways of life and return home with a breadth of knowledge rare at a time when long-distance travel exposed the traveller to considerable danger. Pilgrimage as an anthropological structure gave rise to a rich material heritage in the form of places of workshop, hospitals, accommodation facilities, bridges, etc., and an intellectual heritage of myths, legends and songs. For today's pilgrims, the journey, the track of events and the encounters are as enriching as the aim of the trip itself.
}

Aun cuando podemos afirmar que, con otras expresiones, esta descripción está hablando también de movimiento de personas, encuentro y diálogo, que destacamos como referentes conceptuales acuñados desde la reunión de 1994 en Madrid, lo cierto es que la orientación del Programa Itinerarios Culturales Europeos, que actualmente maneja el Instituto (EICR, por sus siglas en inglés) que lleva justamente ese nombre y que tiene sede en Luxemburgo, es bastante distinta a la patrimonial propiamente dicha, que por su parte alientan UNESCO e ICOMOS.

En efecto, si pensamos en el Camino de Santiago como una ruta continua y con una historicidad y materialidad que la hace susceptible de ser estudiada como un fenómeno concreto, estamos frente al concepto patrimonial. Pero muchas de las rutas incluidas posteriormente como itinerarios europeos por el EICR no tienen estas características, sino que responden a otros criterios como:

- Hacer más visible, valorar más y poner en práctica cotidiana la identidad cultural de los ciudadanos europeos.

- Salvaguardar y enfatizar el patrimonio cultural europeo como factor para mejorar las condiciones de vida como una fuente de desarrollo social, económico y cultural.

- Dar a los ciudadanos nuevas posibilidades de disfrutar a plenitud su tiempo libre otorgando un estatus especial al turismo cultural y sus prácticas sucedáneas.

Pero la idea queda más clara si vemos el concepto oficial de itinerarios culturales europeos, que el EICR (s/f) presenta en los siguientes términos:

By European cultural route one understands a trajectory covering one or more countries or regions, organized around topics whose historical, artistic or social interest proves to be European, either because of the geographical layout of the route, or due to its contents and significance.

Estamos pues frente a un concepto abierto que no responde a la preexistencia histórica de un itinerario cultural en términos concretos, sino que se trata de algo "organizado" en torno a temas de interés histórico, artístico o social, pero que no estuvieron necesariamente históricamente interconectados a través de una ruta concreta.

Lo anterior ha dado lugar a una serie de rutas "temáticas", sin lugar a dudas de interés, y muchas de las cuáles contribuyen al objetivo de fondo del programa, que es crear lo que podríamos llamar "europeidad"; pero que no responden a la investigación científica de un itinerario determinado y concreto. Es cierto que los itinerarios europeos tienen gran inte- 
rés cultural, pero no forman una unidad patrimonial en sí mismos, requisito sine qua non de los itinerarios pensados en los términos formulados por UNESCO e ICOMOS.

La gran pregunta que subyace de lo antes descrito es: ¿Son incompatibles estas dos aproximaciones al uso del término itinerarios culturales? La respuesta que intentaremos defender es: no lo son absolutamente, pues podemos encontrar casos de plena confluencia como en el propio Camino de Santiago y otras rutas europeas como la Ruta Francigena. Por otra parte, aunque son propuestas distintas, resultan igualmente válidas, cada una de acuerdo al contexto institucional y temático al que responden. El problema está simplemente en la necesidad de establecer criterios diferenciales en el uso del término en sí mismo.

Tal vez, como veremos, el giro que se puede ver en el Anexo I de las Directrices Prácticas de la Convención de Patrimonio Mundial de la UNESCO (2012 por su última versión disponible en inglés; 2011 en español), donde se usa el término "itinerarios patrimoniales" ${ }^{1}$, pueda aclarar el punto. Sin embargo, el uso de esta variante terminológica no parece tener éxito en el mundo académico ni en el social, mucho menos en el turístico.

\section{El concepto de "itinerarios culturales patrimo- niales" en las directrices prácticas para la aplica- ción de la Convención del Patrimonio Mundial}

La Convención del Patrimonio Mundial, Cultural y Natural, aprobada por la Asamblea General de la UNESCO en su $17^{\text {a }}$ Reunión celebrada en París (17 al 21 de octubre de 1972) marca un hito en la evolución del concepto y alcances del patrimonio cultural, pero también, y con mayor interés, un proceso. De hecho, la incorporación de nuevas categorías al debate en torno a la Convención, las ha consagrado y dado vigencia mundial.

Veamos previamente cómo es que ese proceso evolutivo se ha ido dando y cómo nos ha acercado al concepto que motiva el presente artículo. Para Francioni (2003), la Convención ha ido incorporando una serie de criterios, haciendo suyo el 'principio de interpre-

1. El término utilizado en inglés es heritage routes, y se hace la aclaración que el término routes equivale a "cultural itineraries", con lo que podríamos decir que estamos frente a los "itinerarios culturales patrimoniales", diferentes por ejemplo a los "itinerarios culturales temáticos europeos". tación evolutiva' propio del Derecho Internacional, por el que la forma en que se interprete el tratado mismo debe considerar y evaluar los desarrollos que desde el Derecho Internacional se han dado en situaciones similares, de preferencia dentro del mismo ámbito. El mismo autor pone como ejemplo de la aplicación del referido principio jurídico, la incorporación del concepto de paisaje cultural a las Directrices de la Convención.

El proceso de evolución y cambio de las citadas directrices es sumamente interesante. Podría dar inclusive la idea de cierta inestabilidad conceptual si sabemos que han sido cerca de 20 las versiones modificadas, algunas en mayor y otras en menor escala, que se han dado de este documento, que podría clasificarse como el "reglamento" de la Convención de Patrimonio Mundial. Sin embargo, justamente esos mecanismos de revisión constantemente atentos son los que han permitido la incorporación de nuevas categorías y principios de gestión de gran utilidad. Un análisis detallado de ese proceso, sobre todo en lo referente a los criterios para la inclusión de nuevos bienes en la Lista y las categorías incorporadas, lo desarrollamos bajo el título Itinerarios culturales y patrimonio mundial (Martorell, 2010).

Sin embargo, el texto del Anexo 3 de las actuales Directrices Prácticas (Directrices para la inscripción de tipos específicos de bienes en la Lista del Patrimonio Mundial) es genérico y hasta cierto punto ambiguo y requiere de una revisión que, seguramente, habrá de suceder en no mucho tiempo dada la cada vez mayor vigencia del tema.

Cuatro son las categorías de bienes patrimoniales a los que se refiere el Anexo 3 comentado. Se trata de los paisajes culturales, las ciudades históricas y centros urbanos, los canales patrimoniales y los itinerarios patrimoniales.

Tal vez debido a la mayor antigüedad de la discusión en torno a los paisajes culturales y sobre todo a las categorías urbanas, los conceptos allí son bastante claros y definen de manera directa el objeto de estudio. Los paisajes culturales recogen en realidad lo conceptualizado en la propia Convención del Patrimonio Mundial cuando en su artículo 1 se refiere a las "obras conjuntas del hombre y la naturaleza"

Sin embargo, en lo relativo a "itinerarios patrimoniales", no se encuentra la misma claridad, tanto así que no hay un concepto propiamente dicho sino 
una serie de lineamientos aproximativos que contribuyen solo parcialmente. Veamos:

El concepto de rutas patrimoniales ha demostrado ser rico y fértil, ya que ofrece un marco privilegiado en el que pueden operar conjuntamente el entendimiento mutuo, un enfoque plural de la historial y la cultura de la paz. (Numeral 23 de las Directrices, 2011).

A lo anterior se agrega que una ruta patrimonial "se compone de elementos tangibles cuyo significado cultural proviene de intercambios y de un diálogo multidimensional entre países o regiones, y que ilustra interacción del movimiento, a lo largo de la ruta, en el espacio y en el tiempo".

A diferencia del tratamiento de las otras categorías, el "concepto" de "itinerarios patrimoniales" no es tal y se encuentra debilitado por ese hecho. Podríamos explicar esto en el relativamente poco tiempo transcurrido desde que el debate se iniciara, pero esto ya no resulta del todo cierto. La gran carencia que hemos señalado en ya numerosas ocasiones radica en no haberse realizado ninguna gran reunión internacional que aúne o por lo menos ponga pautas comunes a los procesos conceptuales en discusión, que ni siquiera se limitan únicamente a lo relativo al marco europeo y de la UNESCO, sino que incluyen casos como los chemins de fer en Francia, el mismo tema ferroviario en Japón, las autopistas escénicas en Estados Unidos y Australia, entre otros.

Los otros aspectos que aborda el Anexo 3 de las Directrices son más específicos al proceso relativo a las candidaturas e inscripción en la LPM, por lo que nos resulta menos interesante. Reproducimos referencialmente el cuadro con el que abordamos el tema (Martorell, 2010, p. 75).

\section{El concepto de "itinerarios culturales patrimo- niales" en la Carta Internacional de Itinerarios Culturales de ICOMOS (2008)}

En la Asamblea General de ICOMOS, celebrada el año 2008 en Quebec, Canadá, se aprobó la llamada "Carta Internacional de Itinerarios Culturales" (2008a), documento que en teoría debería haber puesto punto final al debate académico, por lo menos desde el punto de vista de la vertiente patrimonial. Sin embargo, ese logro no parece haberse alcanzado. El autor de este artículo fue miembro activo del Comité de Itinerarios Culturales, participando en la parte inicial de los debates que llevarían a la redacción del texto que luego aprobaría el
ICOMOS. Pero la tendencia a una visión "clasicista", podríamos decir propia del pensamiento moderno que inspirara la Carta de Venecia en su momento, documento útil pero que en nuestros tiempos requiere ser revitalizado para integrarse, acabó eliminando de los alcances de la Carta Internacional los aspectos más innovadores y que representan los aspectos teóricos más valiosos, de acuerdo con nuestro punto de vista.

Uno de esos alcances había sido expresado en el Anexo 3 de las Conclusiones de la Reunión de Madrid (UNESCO, 1994), donde se consideró como uno de los mayores éxitos de la inclusión del concepto de itinerarios patrimoniales, es que significaría para las comunidades nómadas la posibilidad de ser reconocidas en igual grado que aquellas estables sobre el territorio. Como veremos, la actual Carta de Itinerarios Culturales cierra prácticamente de manera absoluta esa posibilidad.

El concepto de la Carta Internacional comentada (ICOMOS, 2008a) define itinerarios con los siguientes términos:

Toda vía de comunicación terrestre, acuática o de otro tipo, físicamente determinada y caracterizada por poseer su propia y específica dinámica y funcionalidad histórica al servicio de un fin concreto y determinado, que reúna las siguientes condiciones:

- Ser resultado y reflejo de movimientos interactivos de personas, así como de intercambios multidimensionales, continuos y recíprocos de bienes, ideas, conocimientos y valores entre pueblos, países, regiones o continentes, a lo largo de considerables periodos de tiempo.

- Haber generado una fecundación múltiple y recíproca, en el espacio y en el tiempo, de las culturas afectadas que se manifiesta tanto en su patrimonio tangible como intangible.

- Haber integrado en un sistema dinámico las relaciones históricas y los bienes culturales asociados a su existencia.

Expondremos a continuación las principales observaciones al citado artículo de la Carta. De acuerdo con lo antes citado, en primer lugar se trataría de una "vía de comunicación". La primera imagen que se nos viene a la cabeza es la de un trazado físico, una calzada, una autovía, una obra de ingeniería que se extiende sobre el territorio. Es decir, una realidad física concreta, cuya esencia no es solo su materia, sino que además tiene un diseño diferenciable. Así, en un texto 


\section{Cuadro 1. Resumen de los alcances de las Directrices Prácticas respecto a la categoría "itinerarios patrimoniales"}

\begin{tabular}{|c|c|}
\hline Alcances & $\begin{array}{l}\text { Marco para el entendimiento mutuo } \\
\text { Visión plural de la historia } \\
\text { Cultura de la paz }\end{array}$ \\
\hline Componentes & Elementos tangibles \\
\hline Procesos & $\begin{array}{l}\text { Intercambios entre países y regiones } \\
\text { Diálogo multidimensional } \\
\text { Movimientos de interacción }\end{array}$ \\
\hline Extensión & $\begin{array}{l}\text { Continuidad en el espacio } \\
\text { Continuidad en el tiempo } \\
\text { Países o regiones }\end{array}$ \\
\hline Naturaleza & Puede considerarse un tipo dinámico de paisaje cultural \\
\hline Propósito & $\begin{array}{l}\text { Multidimensional, con un propósito principal y adicionales } \\
\text { Religioso } \\
\text { Comercial } \\
\text { Administrativo } \\
\text { Otros }\end{array}$ \\
\hline Valor & Valor de conjunto superior a la suma de elementos \\
\hline Requisitos & Valor universal excepcional \\
\hline Identificación & Basada en fortalezas y elementos tangibles \\
\hline Autenticidad & $\begin{array}{l}\text { Aplicable sobre la esencia del significado del itinerario } \\
\text { Duración de la ruta } \\
\text { Frecuencia en el uso actual } \\
\text { Los legítimos intereses de desarrollo de las poblaciones involucradas }\end{array}$ \\
\hline Contexto & $\begin{array}{l}\text { Marco natural } \\
\text { Dimensión intangible } \\
\text { Dimensión simbólica }\end{array}$ \\
\hline
\end{tabular}

Fuente: Anexo 3 de las Directrices Prácticas (2005). Elaboración propia. 
clásico, Potrykowski y Taylor (1984, p. 11) definen la vía de la comunicación como "la parte del suelo destinada al tráfico de vehículos y, por consiguiente, acondicionada de forma correspondiente", estableciendo como clases de vías: "las de tráfico rodado, acuáticas, férreas". En pocas palabras, un bien material de tipo específico que, aunque sirva para el desplazamiento, es, sin embargo, mucho más típico de culturas de presencia estable en el territorio que las nómadas.

Una ruta puede extenderse básicamente sobre tierra o agua, a lo que la tecnología relativamente reciente nos ha permitido incluir el aire. Pero como contenido material estamos frente a realidades completamente distintas. El componente terrestre es claramente material y concreto: la propia ruta, ya se trate de caminos demarcados por bordos, muros o señales; ya sea de pavimentos de piedra romanos o incaicos, o incluso de las autovías de alta velocidad por las que hoy nos trasladamos. Esta materialidad debe subsistir y, solo por excepción, de acuerdo con la Carta Internacional (ICOMOS 2008a) puede admitirse como patrimonio una vía en gran parte desaparecida, siempre y cuando sus secciones más significativas subsistan.

Las rutas acuáticas, a diferencia de las antes vistas, pueden tener diversos espacios sobre los que discurren: mares, océanos, ríos, canales de diseño humano, lagos, y seguramente los “trazos” serán distintos en cada caso. Aun en caso de ser muy anchos, los derroteros de ríos y canales están marcados por su propia naturaleza. Pero, sobre todo en tiempos de limitados medios tecnológicos (que son los más de nuestra historia), ¿cómo se establece el trazado en términos patrimoniales (que tienden a la localización geográfica específica) en océanos, mares y lagos?

Se entiende que una vía puede haberse trazado sobre tierra o agua. En el caso del recorrido terrestre, este tiene un componente material, concreto, que es la propia ruta, el camino físicamente demarcado. Esto quiere decir que, en principio, desde el punto de vista de la Carta de ICOMOS, la información relativa a un camino cuyas huellas se han perdido no justificaría el empleo del término itinerario cultural. Esto se matiza al admitir en un parágrafo posterior que un itinerario puede seguir vigente, aún cuando parte del trazado original de su ruta se haya perdido, siempre y cuando las partes más significativas se conserven.

En el caso de las rutas acuáticas, como mencionamos antes, existirían algunas alternativas: su recorrido puede hacerse por mares y océanos, por ríos navegables o por canales o cursos de agua manejados por el hombre.

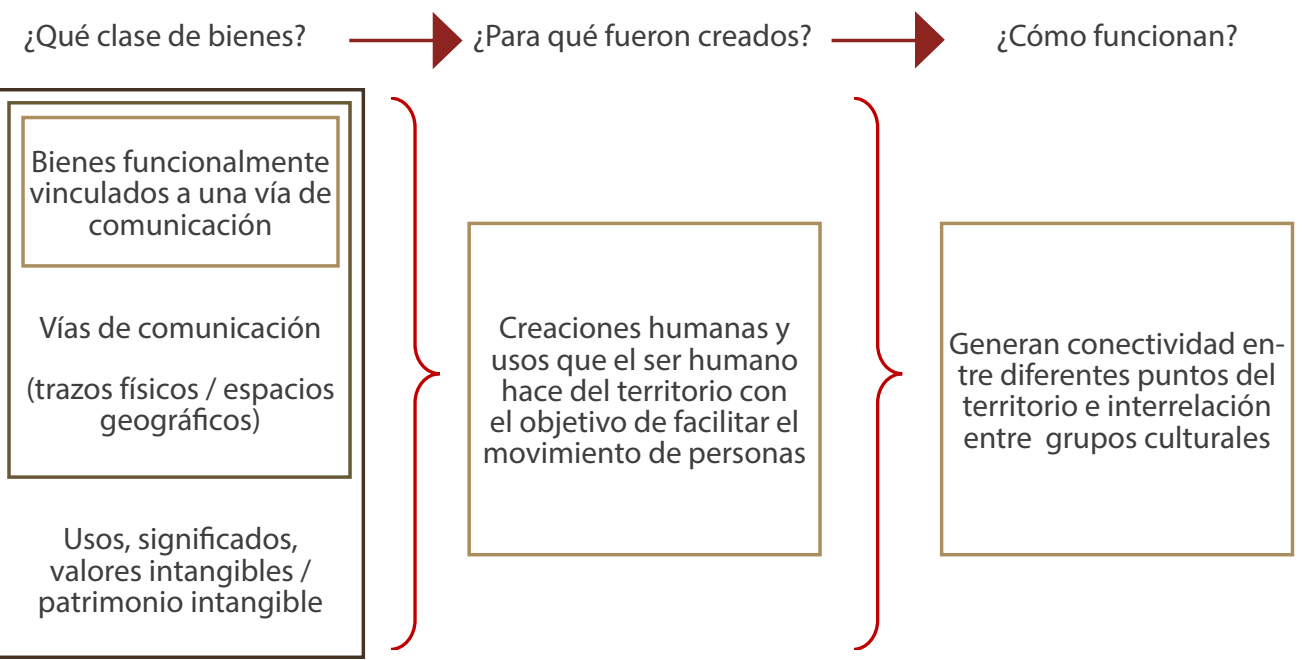

Figura 1

Funcionamiento del concepto de conectividad e interrelación.

Elaboración del autor. 
Los ríos y canales tienen su propio trazo y marco fácilmente distinguible. Para conocer el trazo de una ruta marítima antigua hay que recurrir a otros elementos como los libros de navegación que indican los grandes derroteros seguidos que se pueden calificar como itinerarios, pero con un criterio mucho más flexible, existen los puertos como testimonio físico concreto de los puntos clave, de abastecimiento, protección, comercio y destino de los navíos. Otro posible referente, aunque habría que estudiar científicamente cada caso, son los pecios, que pueden ser testimonios sumergidos de muchas de esas rutas. En los grandes lagos cabría aplicar una lógica similar.

Lo concreto es que en la superficie marítima misma no hay obra física que funcione como elemento facilitador u orientador del derrotero de un barco. Entonces, ¿cómo encaja la exigencia de estar físicamente determinadas, que encontramos en la propia Carta? En algunas superficies terrestres podemos encontrar rutas parecidas que coinciden justamente con las de muchos pueblos nómadas: los recorridos realizados por los desiertos, con "navegantes" cuya huella las condiciones naturales borran, como en el mar, pero como en el mar el hombre ha aprendido a leer otros elementos: los astros, los vientos, los referentes geográficos mínimos. Los puertos en los desiertos son los oasis y son fruto de la obra humana y natural conjuntamente, pero no parecen encajar en el concepto de "vías de comunicación físicamente determinadas". Aunque la analogía con las rutas marítimas podría resultar una alternativa, en ambos casos esa determinación física es solo referencial.

Otro aspecto que nos preocupa del concepto transcrito de la Carta es que se exige que todos los procesos dados a través del itinerario sean vinculables a un fin concreto y determinado. La observación de la realidad nos permitirá determinar que los caminos, aún aquellos consagrados a un fin concreto y determinado, son utilizados para muchos y diversos fines si se quiere, adicionales o incluso secundarios. ¿No enriquece eso el valor del itinerario? ¿No contribuye a explicar la plenitud de su influencia en el territorio por el que se extiende y sus habitantes el conocer $-\mathrm{y}$ reconocer- todas sus funciones históricas $\mathrm{y}$, en su caso, presentes?

Esta exigencia temática se convierte en una nueva cortapisa para un gran número de tipos de rutas. Quedan así fuera del concepto los recorridos de los ferrocarriles, de al menos la mayor parte de las cal- zadas romanas y de las autovías. En el caso de los trenes, para ahondar en un ejemplo, sus posibilidades de reconocimiento se limitarían a las tecnológicas mismas o a su presencia definitoria del paisaje. Esos son valores propios de las rutas de tren, es cierto. Pero su contribución a la historia, al desarrollo, intercambio, comercio, encuentro, transporte y muchos otros procesos humanos es mayor. En Semmering (ferrocarril austriaco inscrito en la LPM en 1998) tenemos la presencia de un tipo arquitectónico desarrollado en una zona que de otra manera se mantendría inaccesible para el ser humano; en los Ferrocarriles de Alta montaña de la India (originalmente inscritos en 1999 y objeto de ampliaciones el 2005 y 2008) podemos encontrar una ocupación del territorio y actividades productivas, como los plantíos de té en las montañas de Darjeeling y Nilgiri, que tienen auge gracias al transporte del producto posible gracias al trazado ferroviario. Pero no solo es la actividad del té, es el tipo de arquitectura al servicio de su funcionamiento: estaciones con sus peculiaridades arquitectónicas, talleres para el mantenimiento de las locomotoras, sistemas de abastecimiento de agua y carbón, sistemas de cremalleras para hacer posible el ascenso en puntos de gran altura, centros poblados crecidos en torno a las estaciones y muchos otros factores, sin los cuales sería imposible explicar la conformación geo-humana de las zonas de influencia de cada uno de estos ferrocarriles. ¿No estamos hablando entonces de procesos de intercambio, interfertilización, valores compartidos y muchos otros que dan mayor valor a los itinerarios como bienes patrimoniales?

Ese es el cambio paradigmático que podrían representar los itinerarios patrimoniales: nos permiten ver ya no solo el bien sino el complejo conjunto de funciones históricas y presentes, surgidos en su entorno no por su simple presencia, sino por su dinámica y funcionalidad. Se pasa de una visión espacial a una funcional que involucra al bien, a su entorno y sus áreas de influencia en sentido amplio. El patrimonio pasa de ser un objeto, a ser un sistema en funcionamiento con gran capacidad de articular territorial, cultural y temporalmente la sociedad.

\section{Buscando criterios objetivos para el replantea- miento del concepto}

Hay algunas características diferenciales que otorgan su significatividad especial a algunos caminos. No es el hecho de que sirvan, como comentamos antes, a 
un fin específico y determinado, aunque en los casos en que se puede determinar un fin principal, como la peregrinación a un lugar sagrado, ese es el leitmotiv que da sentido al itinerario. Todos los caminos implican movimiento de personas, intercambios, influencias mutuas y una serie de otros elementos que hemos venido comentando a lo largo de este artículo. Pero, por supuesto, no todos los caminos se deben o pueden clasificar como patrimoniales. Lo dicho acá es casi una obviedad, pero es necesario partir de ella para preguntarnos entonces por qué, de dos caminos uno es patrimonializable y el otro no.

La respuesta que formulemos a esa pregunta debe ser objetiva, porque busca contribuir a la clasificación científica del patrimonio. Esto tendrá consecuencias concretas en la manera en que entendemos el tema, y nos permitirá desarrollar herramientas de intervención específicas al tipo de bien del que estamos hablando. Es necesario establecer la categoría de protección aplicable al tipo de bien.

García Fernández (2005) señalo al respecto que:

\begin{abstract}
En todo el Derecho, con las categorías de protección se busca atribuir efectos jurídicos y fines a unos objetos materiales. Así se realiza una triple operación (identificar el objeto material, atribuir efectos jurídicos a este objeto y dividirlo en categorías tipológicas). Por consiguiente, la acción de categorizar objetos materiales que constituyen bienes culturales trae causa de la propia variedad material o intelectual de tales bienes y está en relación con el valor social que se le atribuye... La categorización permite precisar el estatuto jurídico de los bienes culturales y afirmar mejor las técnicas de protección y disfrute. Se trata pues del instrumento que permite enlazar las necesidades teóricas de protección de un Patrimonio Histórico dado con las medidas prácticas de protección... (pp. 78-79).
\end{abstract}

Esa fue la tarea que abordamos en nuestra tesis doctoral, sustentada el año 2008 y publicada luego (ver Martorell, 2010). El ejercicio consistió justamente en la búsqueda de los criterios objetivos que podrían ayudarnos a diferenciar primero el tipo de bien cultural qué es un itinerario patrimonial (qué lo diferencia, qué lo caracteriza, qué permite identificarlo) y, luego, orientar el proceso de selección que lleva a elegir determinados itinerarios para que integren el patrimonio, mientras muchos otros quedan fuera.

Un primer derrotero a seguir es la antes mencionada clasificación de ICOMOS (2004), en la que uno de los marcos temáticos es el movimiento de personas dentro del cual se ubican justamente los itinerarios.
La pregunta que entonces nos formulamos es, ¿qué bienes específicamente incluye ICOMOS en la categoría y qué diferencia a esos bienes de otros bienes? En otras palabras, ¿por qué considera ICOMOS que determinados bienes pueden ser diferenciados por su vinculación temática movimiento de personas? Cabe aclarar que para el 2004 había pocos sitios directamente inscritos como itinerarios culturales y que inclusive algunos que lo son, se siguen camuflando como paisajes culturales. La clasificación específica de ICOMOS proponía un total de 111 lugares pertenecientes al marco temático que venimos analizando.

La lectura de los expedientes descriptivos de cada uno de los 111 sitios, nos permitió determinar términos, características y funciones vinculadas con el marco temático, los que ordenamos en el cuadro 2 (Martorell, 2010, p. 182)

De los tres conjuntos propuestos, el A corresponde a bienes concretos, de naturaleza tangible, que tienen un vínculo funcional con vías de comunicación, facilitando en su utilización el movimiento de personas. No se trata de itinerarios culturales, sino de elementos funcionalmente ligados a vías físicas de transporte o a actividades facilitadas por ellas, que podrían ser calificadas como tales. El ejemplo más típico del primer caso son los puentes o túneles, que permiten la continuidad del recorrido ante una barrera natural (quebradas, ríos, montañas). En el segundo caso tenemos centros comerciales, puntos de destino de las rutas mercantiles, centros de peregrinaje, etc.

En el conjunto B encontramos vías de comunicación en sí mismas, es decir, creaciones realizadas con el objetivo $y$, por ende, diseñadas para servir a la movilidad y el transporte. Es importante el hecho de que se trata de bienes cuya razón de ser es facilitar justamente los procesos de movimiento de personas.

El conjunto $\mathrm{C}$ contiene términos que se refieren a actividades humanas que implican el desplazamiento de personas y de bienes a través de mecanismos que, bajo una lógica de funcionamiento en conjunto, constituyen las vías de comunicación. Es el caso, por ejemplo del comercio, actividad que implica la existencia de medios que faciliten el transporte de mercancías, los lugares donde se realizan las transacciones, los de almacenaje, etc. Otro ejemplo de esta naturaleza es el peregrinaje, en el que, en principio, se encuentra el Camino de Santiago de Com- 
postela. El nomadismo y la trashumancia también pueden calificarse dentro de este grupo. Inclusive encontramos que existen referencias directas a procesos históricos de movimiento de personas como a la Rutas de los Esclavos o la Ruta de la Seda.

Identificados los bienes, tipos de bienes y actividades vinculadas al movimiento de personas, el siguiente paso necesario es entender cómo es que funcionan y se relacionan para generar el itinerario patrimonial. Sobre este punto, sin embargo, es necesario que formulemos una aclaración interesante: la mayor parte de casos que ICOMOS clasifica dentro del marco conceptual "movimiento de personas", no están inscritos como itinerarios, sino como bienes puntuales. Lo interesante es que pone en evidencia que entender las relaciones y comunicaciones de estos bienes, enriquece su valía e interés y nos da pautas para su mayor y más preciso conocimiento.

Pero volviendo a nuestra pregunta sobre el funcionamiento y relaciones que los bienes clasificados en los conjuntos $\mathrm{A}, \mathrm{B}$ y $\mathrm{C}$, encontramos un vínculo de pertenencia de los bienes del conjunto A respecto del B y de ambos y en ese orden respecto del C. Es obvio, el puente pertenece a la vía de transporte y ambos son clave para una actividad como el comercio.

Nos preguntamos también sobre la razón de ser de su creación. Siguiendo con el mismo ejemplo, el puente busca salvar el obstáculo natural (quebrada, río, precipicio) para conectar las dos orillas o extremos del mismo y permitir así que se desplacen las personas y bienes. La vía de comunicación busca hacer eso mismo a mayor escala geográfica. Tendrá, por ejemplo, un diseño que permita hacer el camino técnicamente más adecuado, tratará que donde haya inclinación o desnivel este no represente un peligro, etc. En resumen, su finalidad es hacer más fácil el desplazamiento de personas y bienes. Y finalmente los conceptos más abstractos del conjunto $\mathrm{C}$ explican ese mismo fenómeno de movimiento de personas.

De todo lo anterior resumimos nuestra propuesta de que los dos factores diferenciadores de los itinerarios patrimoniales son la conectividad y la capacidad de generar interrelaciones.

La acepción más cercana e inspiradora del uso que acá proponemos para este término la encontramos en el ámbito de los paisajes naturales dentro del campo de la ecología. Se refiere a las relaciones y contactos entre las diversas teselas del territorio. Estas constituyen flujos naturales de energías y nutrientes, movimiento de especies, colonización de territorios por otras especies, etc. Lógicamente, no es a estos procesos, pero sí a los similares que se dan en el reino humano a los que nos referimos. La conectividad de la que venimos hablando se explica en torno a intereses y procesos humanos. Se conectan puntos del territorio que la sociedad y los Estados o los poderes de la administración territorial están interesados en conectar.

Una aproximación a la funcionalidad que la interconexión genera la podemos encontrar en Whebe11 (1969), que hace uso del término corredor como "un patrón lineal de pueblos unidos por rutas de transporte altamente desarrolladas. Comprenden el sistema espacial histórico primario de difusión de la innovación (...)" (p. 4).

Para plantearlo en términos sencillos, el uso que damos a conectividad es el de la acepción más básica que hallamos en el Diccionario (online) de la Real Academia de la Lengua Española: "capacidad de conectarse o hacer conexiones". Utilizando la misma fuente (RAE), encontramos que conexión se refiere, entre otras acepciones, a la concatenación de una cosa con otra y a la acción y efecto de conectar. $\mathrm{Y}$ es justamente buscando el significado de conectar, que encontramos el sentido mayor al uso que damos al término: "Unir, enlazar, establecer relación, poner en comunicación" y "lograr una buena comunicación con alguien".

De lo anterior podemos concluir que al referirnos a conectividad estamos hablando de una capacidad de unir o concatenar partes, de la propiedad de estar conectado y de un sistema, que a su vez implica comunicación, establecimiento de relaciones y flujos materiales e inmateriales.

Nuevamente podemos recurrir al concepto de "conectividad ecológica”. Para Crooks y Sanjaya (2003, p. 6), "At its most fundamental level, connectivity is inherently about the degree of movement of organisms or processes - the more movement, the more connectivity". A lo que agrega posteriormente:

we can identify two primary components of connectivity (Bennett 1999; Tischendorf and Fahrig 2000; Taylor et al. Chapter 2): (1) the structural (or physical) component: the spatial arrangement of different types of habitat or other elements in the landscape, and (2) the functional (or behavioral) component: the behavioral response of individuals, species, or ecolo- 
gical processes to the physical structure of the landscape. Structural connectivity is often equated with the spatial contagion of habitat, and is measured by analyzing landscape structure without any requisite reference to the movement of organisms or processes across the landscape. Functional connectivity, however, requires not only spatial information about habitats or landscape elements, but also at least some insight on movement of organisms or processes through the landscape (p. 3).

La parte funcional, en nuestro caso, la entendemos más en la segunda característica o capacidad de interrelación. La conectividad se refiere sobre todo al componente físico. Los puntos de referencia a ser conectados son los de carácter antrópico (es decir, espacios habitados por el hombre, centros de explotación agrícola, minera, etc., lugares de contenido sagrado, etc.). Es decir que, aunque tomemos la acepción del campo de la conservación de la naturaleza, nosotros estamos hablando de un fenómeno cultural, cuya realización concreta se da a través de construcciones o usos del territorio hechos para permitir que los seres humanos, productos, información, etc., fluyan entre diversos puntos de la geografía humana.

Las vías de comunicación son el tipo básico de bienes que se caracterizan por su propiedad de conectividad. Pero otras creaciones humanas, cuya vocación es hacer posible el flujo de personas y bienes que mantiene viva la vía (estaciones de tren, centros de avituallamiento para los viajeros, tambos incas, fortificaciones, etc.), son bienes que contribuyen al mismo fin, por lo que forman parte de la categoría, por supuesto, de manera asociada a la vía a la que sirven.

Ahora bien, la conectividad que el hombre desea desarrollar tiene por objetivo y consecuencia el encuentro entre seres humanos que ocupan lugares distintos y distantes del territorio. Ese encuentro implica que unos y otros usuarios de un camino se interrelacio-

Cuadro 2. Clasificación de los elementos vinculados al tema movimiento de personas

\begin{tabular}{|c|c|c|}
\hline Conjunto A & Conjunto B & Conjunto $\mathrm{C}$ \\
\hline $\begin{array}{l}\text { Bienes tangibles funcionalmente vin- } \\
\text { culados a una vía de comunicación }\end{array}$ & $\begin{array}{l}\text { Vías de comunicación (Trazos físicos/ } \\
\text { espacios geográficos) }\end{array}$ & $\begin{array}{l}\text { Usos, significados, valores intangibles / } \\
\text { patrimonio intangible }\end{array}$ \\
\hline Puentes & Viaductos & Caravanas \\
\hline Estaciones de tren & Canales & Rutas de los esclavos \\
\hline Oasis & Itinerarios & Comercio \\
\hline Túneles & Carreteras & Nomadismo \\
\hline Centros de comercialización & Vías & Trashumancia \\
\hline Centros de peregrinaje & Redes (de transporte) & Transporte \\
\hline Puertos & Ríos & Comercio \\
\hline Puntos comerciales & Ferrocarriles & Tráfico \\
\hline Centros comerciales & & Inmigración \\
\hline \multirow[t]{8}{*}{ Edificios comerciales } & & Peregrinaje \\
\hline & & Navegación \\
\hline & & Itinerario \\
\hline & & Sistema de comunicación \\
\hline & & Sistema de transporte \\
\hline & & Distribución \\
\hline & & Ruta de la seda \\
\hline & & Transporte acuático \\
\hline
\end{tabular}

Elaborado en base a los términos tomados de los expedientes para la inscripción en la Lista del Patrimonio Mundial de los bienes que el ICOMOS ha calificado en cada una de las subdivisiones del marco temático movimiento de personas. 
nen entre sí. El Diccionario de la RAE define interrelación como "Correspondencia mutua entre personas, cosas o fenómenos".

La "correspondencia", que nosotros llamaremos encuentro e intercambio entre personas, genera intercambio de valores, conocimientos, creencias, puntos de vista, forma de entender el mundo, usos lingüísticos, culinarios, etc. Hablamos de todos aquellos planos de interrelación posibles entre dos culturas total o relativamente distintas: sociales, económicos, culturales, espirituales, políticos, religiosos, tecnológicos, científicos, etc.

El estudio histórico de un itinerario también nos permitirá, en muchos casos, entender que estamos frente a la "correspondencia mutua entre cosas o fenómenos". Los estilos arquitectónicos, sus formas de extensión territorial, sus usos, demuestran influencias recibidas a través del camino. Inclusive la presencia de materiales, y no solo los constructivos, sino decorativos y simbólicos, se explican en encuentros e intercambios que la ruta facilitó. El avance de las técnicas y las ciencias, en el larguísimo periodo anterior a los mass media y con mayor razón a las comunicaciones virtuales, se daba a través de los viajeros que, en su memoria o en lo poco comunes libros, fueron extendiendo el conocimiento a la vera del camino, etc.

\section{Casos aplicativos del concepto itinerario patri- monial de data reciente}

Aun cuando ha habido claras resistencias a la aceptación del concepto de itinerarios culturales o patrimoniales, lo cierto es que en los últimos tiempos este campo viene adquiriendo mayor autonomía y reconocimiento propio.

Aunque el expediente de inscripción como patrimonio mundial del Ferrocarril Raetiano en los paisajes de Albula/Bernina (bien transfronterizo entre Italia y Suiza inscrito en 2008) haga incidencia en el tema paisajístico, nosotros sustentaremos que se trata de un caso de itinerario patrimonial (o cultural).

La descripción oficial del bien en el sitio web del Centro de Patrimonio Mundial de UNESCO es la siguiente:

Este sitio agrupa dos líneas ferroviarias históricas que cruzan los Alpes suizos por dos puertos de montaña. Inaugurada en 1904, la línea del Albula está situada en la parte nordeste del sitio y tiene $67 \mathrm{~km}$ de longitud. Comprende un conjunto impresionante de obras de ingeniería formado por 42 túneles y galerías cubiertas y 144 puentes y viaductos. La línea del Bernina tiene $61 \mathrm{~km}$ y cuenta con 13 túneles y galerías y 52 puentes y viaductos. El sitio es un ejemplo notable de la utilización del ferrocarril, a principios del siglo XX, para acabar con el aislamiento de las poblaciones de los Alpes centrales e inducir un cambio socioeconómico duradero en la vida de los montañeses. Este ferrocarril es una realización excepcional en el plano técnico, arquitectónico y ambiental, que encarna los logros que pueden alcanzar la arquitectura y la ingeniería civil en perfecta armonía con el paisaje circundante.

Podemos encontrar en la descripción anterior el tipo de bienes del que venimos hablando y clasificando. Pero lo que más interesante nos resulta es que de esa manera se logró acabar con el aislamiento de las poblaciones de los Alpes centrales, agregándose a ello que se indujo a un cambio socioeconómico duradero en la vida de los montañeses. Se trata, sin duda, de los dos caracteres diferenciales de los itinerarios que hemos discutido anteriormente con bastante detalle: conectividad e interrelación.

Esto lo podemos ver aún con más claridad en los argumentos expresados para el reconocimiento de los Valores Universales Excepcionales del bien que obran también en la página de internet del Centro de Patrimonio Mundial:

The railway's socio-economic consequences were substantial and lasting for mountain life, the interchange of human and cultural values, and changes in the relationship between man and nature in the West. The Rhaetian Railway offers a wide diversity of technical solutions for the establishment of the railway in often severe mountain conditions. It is a well designed construction that has been realised with a high degree of quality and it has remarkable stylistic and architectural homogeneity. The railway infrastructure moreover blends in particularly harmoniously with the Alpine landscapes through which it passes.

En cuanto a la definición como paisaje cultural o como itinerario patrimonial sostenemos que esta última es la coherente, porque es la que abarca el sentido de los procesos de intercambio de valores, reúne una serie de elementos tecnológicos destinados a conectar el territorio y explica los procesos históricos humanos generados por el bien. En cambio, como se puede apreciar en los propios textos citados, la relación con el paisaje de entorno es armónica. Debemos definir el bien por sus características intrínsecas $\mathrm{y}$ no por sus relaciones. 
Un factor que se requiere tener en cuenta para entender la visión distinta que implica la conservación y gestión de itinerarios culturales es que no se trata de un bien simple y aislado, sino interconectado y potenciado en valor -aunque al mismo tiempo en complejidad de gestión- por los diversos tramos de su recorrido y los numerosos bienes que lo conforman. Dentro de esos, hay algunos que tienen una importancia mayor, porque están directamente vinculados a lo lógica funcional y sistémica del itinerario.

En el caso de Albula / Bermina, el informe técnico de ICOMOS (2008b) se refiere a una serie de elementos técnicos, de ingeniería y de recorrido mismo de la línea férrea, pero también incluye:

The nominated properties in the immediate environment of the line: these are the built structures used for railway functions, consisting in particular of the stations, of which there are 36 , and their facilities (platfor$\mathrm{ms}$, sheds, etc.). There is great stylistic diversity, partly as a result of the many restorations, reconstructions and extensions. To take one example, the station of Célerina has been rebuilt or extended on four occasions since its initial creation in 1903. (p. 208).

Este tipo de instalaciones y construcciones forman parte del núcleo central a proteger, porque sin ellos sería imposible que el ferrocarril se hubiera mantenido en funcionamiento hasta nuestros tiempos. Hay alguna otra mención a elementos fundamentales, como la estación eléctrica de Campocologno que proveyó de electricidad a la línea de Bernina. Sin embargo, se puede observar que no se especifican otros elementos vitales para el funcionamiento de este medio de transporte, como los talleres, los puntos de abastecimiento de agua, etc.

Los llamados Ferrocarriles de Alta Montaña de la India, han sido materia de ampliaciones de su inscripción inicial, convirtiéndose en un bien seriado (aquel que, si bien no tiene continuidad geográfica o física, sin embargo se entiende como parte de una unidad histórica, estilística, etc., y por ende se trata unitariamente). El primer recorrido en ser inscrito, en 1999, fue el de la mítica Darjeeling, la Montaña Azul que tanto ha despertado la imaginación del mundo occidental. Posteriormente se han agregado los recorridos de tren en la Montaña de Nilgiri, en el Estado de Tamil Nadú, al sur de la India y de Kalka-Shimla y en el Estado de Himachal-Pradesh.

Si bien uno de los factores más resaltados, y que merece serlo, es el avance tecnológico que la construc- ción de estos tramos ferroviarios significó, también es cierto que existen una serie de otros valores que igualmente contribuyen a la plenitud del bien. Así, el sitio web de UNESCO, en su descripción larga, dice: "The Darjeeling Himalayan Railway is intimately linked with the development of Darjeeling as the queen of hill stations and one of the main teagrowing areas in India, in the early 19th century." En el caso de la montaña de Nilgiri, igualmente famosa por su producción de té, la llegada del ferrocarril significa también un cambio trascendental en toda su configuración socioeconómica y, por ende, su devenir histórico. Aislar la comprensión de los trenes a solamente su valor tecnológico, es dejar de lado otros factores que bajo la perspectiva de los itinerarios patrimoniales se pueden poner en evidencia.

El caso más reciente de bien incluido en la LPM que se puede tipificar como itinerario patrimonial, es el del mexicano Camino Real de Tierra Adentro, inscrito en 2010.

Haremos un paréntesis para referirnos a los dos criterios, bastante diferentes, que se han seguido al momento de inscribir itinerarios en la LPM. El primero corresponde al Camino Francés, (el Camino de Santiago en España), único bien que ha sido inscrito de manera integral en sus cerca de $800 \mathrm{~km} \mathrm{y}$ numerosísimos bienes asociados. La otra alternativa, seguida tanto en el caso del Camino de Santiago en Francia como en el de Tierra Adentro, es seleccionar determinados tramos a los que se considera representativos del conjunto y que se inscriben bajo la ya mencionada modalidad de bienes seriados.

El informe de ICOMOS (2010) sobre el Camino Real de Tierra Adentro precisa:

The nominated property is a series of 60 separate sites along or near a $1,400 \mathrm{~km}$ stretch of the Camino Real de Tierra Adentro. Also known as the Silver route, this road was developed primarily for the export of silver to Spain from the mines of Zacatecas, Guanajuato and San Luis Potosí, north-west of Mexico City, and the import of mercury from Spain and at time Slovenia. It was a part of the Intercontinental Royal Road that linked Mexico City to Veracruz on the coast in the south and thence to Spain. It was also part of what is now called the Mercury route between Spain and the three main silver mines Initially the Camino Real de Tierra Adentro ended at the Zacatecas, it was then extended northwards to Santa Fe, New Mexico.

The full $2,600 \mathrm{~km}$ extent of the route thus embraces Hispanic founded towns in Texas and New Mexico, in 
territory now known as the United States of America. Sites along this latter stretch of the route have not been nominated, but the nomination suggests will be in the future. (p 100).

La cita amplia es necesaria porque nos permite una idea de la extensión del bien, que estaría representado por algunos de sus tramos. En teoría, el sistema de protección ideal sería el del Camino Francés (el Camino de Santiago en España), donde la integridad está garantizada. En la práctica, empero, hemos visto cómo en numerosas ocasiones los criterios de conservación chocan con necesidades como la construcción de grandes autopistas que implican variaciones incluso en el trazado del camino. Desde ese punto de vista, puede ser necesario ceder en algunos aspectos ideales a cambio de logros más bien prácticos. Pero hay que ser sumamente cuidadosos con la representatividad real de los tramos elegidos y con la concepción de la funcionalidad integral de la ruta que en muchas ocasiones no responde a criterios estéticos ni monumentales. No necesariamente los tramos espectaculares, las obras maestras en torno a la ruta y otros elementos similares son los que representan al bien.

En el Camino de Santiago hay un ejemplo altamente significativo de lo que estamos tratando de explicar. En la provincia de León, ya cerca a los límites con la Comunidad Gallega donde se encuentra Santiago de Compostela, en el lugar llamado Monte Irago se encuentra una sencilla Cruz de Fierro (Cruz de Ferro en gallego), erigida sobre un simple trozo de madera de algo de 5 metros, y debajo de la cual se encuentra un cada vez mayor montículo de piedras que a su paso los peregrinos van dejando como ofrenda o como símbolo de su peregrinar. Sobre este sitio hay diversos escritos, y no queremos profundizar en sus intepretaciones. Pero lo que queda claro es que es un hito profundamente simbólico para el caminante quien, además, en caso de estar enfermo recibirá las indulgencias plenas (que corresponden al peregrino que llega hasta Compostela en Jubileo) a poca distancia, en un bello pueblo llamado Villafranca del Bierzo, exactamente en su Iglesia de Santiago. Ese sencillo hito no tiene punto de comparación con la maravilla arquitectónica de catedrales como las de Burgos, Astorga, León o Santiago mismo. Pero para la protección del camino, si hubiera un tramo que elegir, el de Monte Irago sería uno de los más importantes.

En el caso de Tierra Adentro, los sitios elegidos comprenden 11 centros o conjuntos históricos, 9 pueblos, 7 antiguas haciendas, 6 capillas, 5 puentes separada- mente y dos asociados con otros sitios, 4 tramos de camino, 2 antiguos colegios, 2 cuevas, una mina, un antiguo convento y un cementerio. En su trazado se encuentran también el Centro Histórico de Ciudad de México, la Zona de Monumentos de Querétaro, el Pueblo de San Miguel y el Santuario de Jesús Nazareno de Atotonilco, el Poblado Histórico de Guanajuato y sus minas adyacentes; y el centro histórico de Zacatecas, todos ellos inscritos en la LPM (ICOMOS, 2010). La importancia de los sitios mencionados, sobre todo desde el punto de vista monumental, está fuera de discusión. Pero extrañamos la referencia específica a, por ejemplo, las postas que se extendían a lo largo de este tipo de caminos y que permitían el desplazamiento de las personas que, sin al menos fuentes de agua y lugares para descansar junto con sus caballos o ganado, podrían perecer en el intento de viaje. Al menos los más representativos de esos elementos, no necesariamente grandiosos pero sí funcionalmente significativos, deben ser conservados dentro del bien principal. Cada camino tiene su lógica funcional: en el caso de los trenes, los depósitos de agua y carbón están ubicados a una distancia que permite el recorrido sin riesgo a quedarse en medio viaje por falta de estos elementos. En los desiertos, la lógica de los ojos de agua y oasis, obligará al viajero a establecer un ritmo y llevar una cantidad de provisiones, pero también a modificar y crear incluso oasis artificiales que garanticen su supervivencia. En los caminos de herradura, las postas donde hombres y animales descansen, se alimenten y reciban las atenciones necesarias, son funcionalmente indispensables para que el movimiento de personas sea o haya sido posible.

Podemos volver al Camino de Santiago para hacernos una idea más clara de lo que venimos sosteniendo: los hospitales de peregrinos son simbólica y físicamente hitos indispensables si queremos entender la lógica del funcionamiento del itinerario de peregrinaje. Ubicados estratégicamente, brindan refugio y pan desde tiempos medievales a los peregrinos. Sin ellos, las posibilidades de sobrevivir en el viaje, en tiempos medievales, eran mínimas. Si se tiene que priorizar qué bienes consagrar en el grado más alto de protección, esos hospitales son fundamentales.

La lectura de los bienes que forman parte de las zonas especialmente inscritas en la Lista del Patrimonio Mundial en el mexicano Camino Real de Tierra Adentro, no parece haber prestado mayor atención a esos elementos ligados al funcionamiento mismo del camino como tal. 


\section{El Qhapaq Ñan: en la ruta a ser declarado Patri- monio Mundial}

La vigencia del tema de los itinerarios culturales en nuestra región y tiempos está en un grado alto porque nuestro territorio y el de 6 de los países de nuestro subcontinente, constituyen el medio geográfico donde se ha desarrollado uno de los mayores y más impresionantes itinerarios patrimoniales del mundo entero. Hoy llamado Qhapaq Ñan, Camino Principal Andino, se trata del conjunto de vías que fueron diseñadas durante el desarrollo de la cultura Inca, que habría integrado rutas de pueblos que ocuparon antes el mismo territorio, dándoles, sin embargo, una coherencia de proceso político-administrativo que lleva a arqueólogos de la talla de Luis Guillermo Lumbreras a calificar este extenso bien justamente como el gran proyecto político de los incas.

De acuerdo con el Proyecto Qhapaq Ñan (Ministerio de Cultura del Perú, 2013), se trata de "una extensa red de caminos formalmente usada por los incas en el siglo XV, que tuvo como objetivo articular los diversos pueblos del Tawantinsuyu para una eficiente administración de los recursos existentes a lo largo del territorio" (p. 5).

Los bienes cuya candidatura a la LPM es presenta$\mathrm{da}$, son evaluados en base a una serie de criterios contenida en el parágrafo 77 de las Directrices de la Convención de Patrimonio Mundial. De acuerdo con el documento antes citado, el Qhapaq Ñan reúne al menos los 6 criterios culturales. Es probable también que, en determinados tramos, existan suficientes valores naturales como para agregar a ese listado alguno de los criterios propiamente naturales. Sin embargo, la clave no está en la demostración de que los reúne, sino en la comprensión de cómo se aplican ante una categoría compleja de bien como es un itinerario cultural.

Indiscutiblemente las condiciones actuales del subcontinente harían absolutamente inviable conservar, dentro de los rigurosos alcances de la gestión de bienes del patrimonio mundial, un bien que cruza completamente hasta 3 países sudamericanos (Perú, Bolivia y Ecuador), alcanza grandes proporciones de otros dos (Argentina y Chile) y llega hasta un sexto, que es Colombia. Pero es de desearse que en los criterios de priorización de qué tramos se van a consagrar al máximo nivel, hayan estado pre- sentes aquellos que nos ayudan a entender la lógica funcional de esta importantísima ruta.

Así por ejemplo, la determinación de los valores de conjunto debe ser la prioritaria. Un itinerario patrimonial es un bien unitario complejo, no varios bienes distintos. Por lo tanto, los valores representados por un elemento en un lado del camino, son igualmente valores compartidos con otros tramos aunque parezcan muy distintos y alejados.

Ahora bien, hay una gran responsabilidad moral al momento de decidir qué tramos se incluyen, que es igual a decidir cuáles quedan fuera de las posibles ventajas del hecho de pertenecer directamente a la LPM. Si el itinerario en sí mismo es una unidad, pero por razones prácticas nos vemos obligados a elegir determinados segmentos como los más representativos, debemos también saber generar mecanismos compensatorios y de equilibrio a favor de los pobladores de las secciones que resultan excluidas.

Es necesario, entonces, ya de cara a la gestión futura del Qhapaq Ñan, desarrollar instrumentos inclusivos que vayan más allá de los límites de los tramos elegidos en la seriación presentada ante la UNESCO. Entre los posibles mecanismos para alcanzar este objetivo, está la construcción y diseño de centros de interpretación ubicados de tal forma que permitan, tanto al poblador local como al visitante, entender que cada sector del camino es parte integrante de un conjunto unitario. Una política coherente en ese sentido podría inclusive permitr, una ampliación del área declarada a otros tramos, de tal modo que los beneficios generados se extiendan. Esto, desde luego, sin descuidar que cada posible tramo nuevo debe estar en condiciones de ayudarnos a entender la funcionalidad del itinerario en sí mismo. Otro mecanismo es crear una figura especial de "zona de amortiguamiento" que, vía una abstracción, se aplique al menos a una gran parte de los tramos que no formen parte de la inscripción inicial en la LPM. Así, dichos tramos gozarían de un sistema que, sin aplicar los criterios más estrictos de conservación, garanticen la subsistencia de valores mínimos que contribuyan a la creación de alternativas de desarrollo en torno al uso sostenible del conjunto. Es más, un sistema de control de efectos no deseados sobre los tramos de mayor representatividad, consistirá en el incentivar también la visita de otros tramos que también forman parte del Sistema Víal Andino. 


\section{Bibliografía}

Crooks, K. \& Sanjaya, M. (eds.). (2006). Connectivity conservation: maintaining connections for nature. Recuperado el 25 de abril de 2013 desde http://www. cambridge.org/servlet/file/store6/item5726114/ version1/9780521857062_excerpt.htm

Consejo de Europa. (s.f.). The Santiago de Compostela Pilgrim Routes. Recuperada el 4 de mayo de 2013 de http://www.coe.int/t/dg4/cultureheritage/culture/routes/compostella_en.asp?

European Institute of Cultural Routes. (s.f.). The Cultural Routes Program of the Council of Europe. Recuperado el 20 de abril de 2013 de: http:// www.culture-routes.lu/php/fo_index.php?lng=en\&dest=bd_do_det\&id=00000263

Francioni, F. (2003). Treinta años después: ¿está la Convención sobre el Patrimonio Mundial preparada para el siglo XXI?. Patrimonio Cultural y Derecho. No 7. BOE-AENA-Hispania Nostra (pp. 11-38).

García Fernández, J. (2005). La función del derecho en la protección de los bienes culturales. En G. Ruiz-Rico \& N. Pérez (eds.). Constitución y cultura: retos del Derecho Constitucional del Siglo XXI (45-80). Jornadas celebradas en la Universidad Internacional de Andalucía. Tirant lo Blanch: Valencia.

ICOMOS (1993). Advisory Body Evaluation. The Route of Santiago de Compostela. (Informe Nro. 669). Recuperado el 5 de mayo de 2013 desde http://whc.unesco.org/archive/advisory_body_evaluation/669. pdf.

ICOMOS (2004). The World Heritage List: Filling the Gaps - an Action Plan for the Future. An Analysis by ICOMOS. Recuperado el 3 de abril de 2013 desde http://whc.unesco.org/uploads/activities/documents/activity-590-1.pdf.

ICOMOS (2008a). Carta Internacional de Itinerarios Culturales. Recuperado el 4 de mayo de 2013 desde http://www.international.icomos.org/charters/ culturalroutes_sp.pdf.
ICOMOS (2008b). Advisory Body Evaluation. Rhaetian Railway (Switzerland / Italy) (Informe Nro. 1276). Recuperado el 18 de abril de 2013 desde http:// whc.unesco.org/archive/advisory_body_evaluation/1276.pdf.

ICOMOS (2010). Advisory Body Evaluation. Camino Real de Tierra Adentro (México) (Informe Nro. 1351). Recuperado el 18 de abril de 2013 desde http:// whc.unesco.org/archive/advisory_body_evaluation/1351.pdf.

Martorell, A. (2010). Itinerarios culturales y patrimonio Mundial. Lima: USMP.

Ministerio de Cultura del Perú (2013). Qhapac Ñan. Sistema Vial Andino. Camino a Patrimonio Mundial. Lima: Ministerio de Cultura.

Potrykowski, M. \& Taylor, Z. (1984). Geografía del Transporte. Barcelona: Ariel.

Real Academia Española (2001). Diccionario de la lengua española (22.a ed.). Consultado en http://www.rae. es/rae.html.

UNESCO (1994). Routes as Part of Our Cultural Heritage. Report On The Meeting Of Experts. Madrid 24-25 November 1994. (Informe Nro. WHC-94/CONF.003/ INF.13) Recuperado el 26 de abril de 2013 de: http://whc.unesco.org/archive/routes94.htm.

UNESCO (2011). Directrices Prácticas para la aplicación de la Convención del Patrimonio Mundial. Recuperado el 12 de abril de 2013 desde http://whc.unesco. org/archive/opguide08-es.pdf.

Whebell, C. F. J. (1969). Corridors: A theory of urban systems. En Annals of the Association of American Geographers. Vol 59, № 1, marzo de 1969. pp 1-26.

Web del Centro de Patrimonio Mundial. http://whc.unesco.org.

Web del Instituto Europeo de Itinerarios Culturales: http://www.culture-routes.lu/php/fo_index. php?lng=en. 